\author{
Alain Di Gallo \\ Felix Amsler \\ Charlotte Gwerder \\ Dieter Bürgin
}

\section{The years after: a concept of the psychological integration of childhood cancer}

Received: 7 July 2003

Accepted: 11 July 2003

Published online: 26 August 2003

(C) Springer-Verlag 2003

\author{
A. Di Gallo ( ) F. Amsler · C. Gwerder \\ D. Bürgin \\ Kinder- und Jugendpsychiatrische \\ Universitätsklinik und -poliklinik, \\ Schaffhauserrheinweg 55, \\ 4058 Basel, Switzerland \\ e-mail: alain.di-gallo@unibas.ch \\ Fax: +41-61-6852178
}

\begin{abstract}
Goals of work: To define and measure the psychological integration of childhood cancer experiences into a personal biography and to explore the association between integration with illness-related factors and psychosocial conditions. Patients and methods: Analysis of cancer survivors' narratives on the course of their illness was used to measure integration. Psychosocial condition, body concepts, health locus of control, and illness-related distress were evaluated by questionnaires. Illness factors were assessed by reviewing hospital case notes and sociodemographic factors by a structured interview. Of 72 eligible subjects contacted, 60 agreed to participate. Main results: High inter-rater correlations established the reliability of the concept of testing integration by narrative analysis. Subjects with good psychological integration of the experience of cancer saw
\end{abstract}

chance as having less to do with illness and health, and perceived illness and therapy retrospectively as more distressing than survivors with poor integration. In contrast, integration did not correlate with distress evoked by present feelings toward illness and therapy or by thoughts of a relapse. Conclusions: Successful integration of the experience of cancer may be associated with the ability to accept painful feelings and to allow them to emerge, and with a readiness to accept responsibility in relation to health and medical care. Assisting young cancer patients and their families to create and maintain their personal narratives of the experience of illness is an important clinical task for all professionals working in paediatric oncology.

Keywords Cancer - Children . Coping $\cdot$ Late effects $\cdot$ Narrative . Survival

\section{Introduction}

Improvements in survival rates over the past few decades have stimulated a growing interest in the emotional and social consequences of childhood cancer. So far, most studies in this area have used interviews and questionnaires with predominantly psychometric characteristics, investigating psychopathology $[1,2,3,4]$, self esteem $[2,3,5]$, body image $[6,7]$ or coping $[6,8,9,10,11]$. Recent reviews have indicated that most survivors show good psychosocial adjustment compared to healthy controls $[12,13]$. However, the experiences associated with childhood cancer are unique, and too complex to be reduced exclusively to comparisons with healthy people using standardized measures. More qualitative approaches may help to provide a more complete understanding of the subjective aspects of illness and survival $[13,14]$.

Only a few investigations have adopted this focus [2, 15]. Boman and Bodegard [9, 10] have investigated patients' coping with illness and treatment using semistructured interviews with survivors of childhood cancer. In their study, good coping was defined as a result of the successful integration of psychic trauma. This approach indicates that stressful experiences must be integrated in- 
to the course of personal biography. If they are not, they must be split off from conscious awareness and voluntary control, a psychic process first systematically described as dissociation by Janet [16]. Janet's definition of dissociation has also been the subject of contemporary research, which has emphasized the disruption of both psychological and physiological integrative functions [17]. While dissociation of negative emotions may reduce the ability to experience any feeling, giving meaning to distressing situations has been associated with a sense of coherence and a positive psychological state $[18,19]$.

According to Janet's theory, a stressful experience becomes traumatic if the person involved, overwhelmed by emotions, loses the ability to create and maintain an integrative story, which he termed "narrative memory" [20]. Narrative construction and retelling are cognitive processes that allow individuals to experience life as a whole and not just as an unrelated series of disconnected events [21]. Narrative is a human resource for creating meaning and integrating past and present in anticipation of the future [22]. Personal narratives help people to organize experience and make sense of stressful life events. Coherent narratives contribute to the sense of identity and may facilitate coping [23, 24, 25, 26], whereas a fragmented life story, not experienced as followable and coherent, may reflect vulnerability and trauma [27]. While questionnaires and structured interviews bear the risk of asking for the expected and neglecting the unusual, narratives urge the listener to follow the course of events in the way the narrator experienced them as relevant and significant [28].

In this study, adult childhood cancer survivors' narratives of the course of their illness were analysed to assess the degree to which the experience of cancer was integrated into personal biography. This exploratory investigation was not aimed at presenting a valid concept of the term "integration", but at evaluating and discussing patients' ability to organize their memories of illness and therapy and "to create a whole out of fragmented parts". The concept of integration covers the following areas:

- Coherence

- Emotionality

- Readiness and ability to recognize the feelings associated with the illness and its significance for the personal life-history

- Appropriate affective distance in the recounting, without either rigid defence or excessive disquiet or fear

The hypothesis is that good integration of the illness into a coherent sense of self, assessed by the content and structure of the subject's talk about their experience, is associated with a positive psychological outcome. The following questions were addressed:

1. Can a concept of "integration of cancer experience", as defined for this study, be reliably measured by narrative analysis?
2. How are the experiences of childhood cancer psychologically integrated into personal biography?

3 . What factors are correlated with integration?

4. Is there an association between integration and present psychological condition?

\section{Patients and methods}

\section{Participants}

The study sample consisted of subjects who (1) had been treated for cancer during childhood or adolescence at the University Children's Hospital, Basel, (2) had finished therapy at least 5 years previously and were in remission, and (3) were 18 years of age or older at the time of participation in the study. A review of the hospital case notes of all former patients found 72 subjects who met the inclusion criteria. These 72 subjects were contacted and $60(83.3 \%)$ agreed to take part, of whom 35 were male and 25 female. Fourteen diagnoses were represented. Of the 60 participating patients, $27(45.0 \%)$ had had acute lymphocytic leukaemia, $6(10.0 \%)$ Hodgkin lymphoma, and the remainder acute myeloid leukaemia, non-Hodgkin lymphoma or solid tumours, and 11 (18.3\%) had suffered relapses. The mean age at diagnosis was 8.5 years (range $0.3-18.4$ years), and the mean age at study participation was 26.2 years (range 18.0-40.5 years). Non-participation in the study was most often due to the desire not to be reminded of distressing memories or because of having "closed this chapter of life". There were no significant differences between participants and non-participants in terms of sex, age, diagnosis or physical after-effects.

\section{Measures}

The key variable "integration" was assessed by means of a narrative interview. Participants were given the following instruction by the interviewer (A.D.G.): "We are interested in how you experienced your illness and whether this has had any effects on the rest of your life. We would like you to tell your story." Sessions lasted 20-70 min and were videotaped for subsequent coding. The narrative was not interrupted by the interviewer unless a series of significant topics (e.g. particularly stressful or helpful experiences) went unmentioned or clarification was necessary. Thus, the aim was not primarily to obtain a complete and structured summary of the course of the illness, but to learn what the survivor considered worth telling and how. The method of narrative analysis used in this study is a modified version of a coding system employed by Oppenheim et al. [29] who investigated the associations between couples' narratives about the birth of their child with their marital satisfaction and psychological well-being.

Seven criteria were used to measure integration:

1. Story coherence (rich and fluent narrative vs fragmented narrative where the listener had to fill in the gaps in order to understand it)

2. Range of emotions (wide range of differentiated emotions vs absence, or narrow and limited range, of emotions)

3. Communication about internal states and processes (good articulation of inner world vs little insight into emotions and personal meaning)

4. Congruence of emotions and contents (high vs low correspondence between the story's content and the emotions expressed)

5. Uni-vs multidimensional illness description (ability to accept different aspects of the experience, positive and negative, and ability to accept ambivalence)

6. Personal significance of illness (understanding and acceptance of personal meaning vs denial) 
Table 1 Coding scale for the criterion personal significance of illness

1 The subject does not consider the illness to have had any influence on the course of his/her further life. He/she adheres rigidly to the idea that development was unaffected, and the accounts of the illness appear erased from personal experience and from individual biography

2 Only a few aspects of the illness are considered in terms of their significance for the personal life history. The ability and willingness of the subject to accept these aspects as part of his/her later development is minimal

3 The narrative contains areas whose significance for the biography is considered and experienced, as well as those that remain emotionally split off and uncomprehended

4 The subject essentially accepts and understands the emotional significance of the experience of illness for his/her further development. Some aspects, possibly the more distressing ones, are excluded from this understanding and remain inaccessible to personal experience

5 The subject is able to deal in an ongoing way with the effect of the illness on his/her life. The significance of the associated experiences is acknowledged, and they form part of the total experience. The value of the illness experience

for the individual biography can be conveyed to the observer in an empathetic way

Table 2 Narrative coding: inter-rater correlations $(n=60)$

\begin{tabular}{ll}
\hline Criterion & Intraclass correlation \\
\hline Story coherence & 0.80 \\
Range of emotions & 0.69 \\
Communication about internal states & 0.76 \\
Congruence of emotions and content & 0.62 \\
Dimensions of illness description & 0.52 \\
Personal significance of illness & 0.64 \\
Interview distress & 0.63 \\
Summary integration scale & 0.79 \\
\hline
\end{tabular}

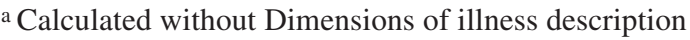

7. Interview distress (extent of distressing emotions evoked by telling the narrative)

The narratives were scored independently by two trained raters (A.D.G., C.G.) using a five-point scale, with high scores indicating better integration. Table 1 shows the coding scale for the criterion personal significance of illness. Inter-rater differences of one point were averaged. In case of greater differences the videotape was discussed by the two raters to find a consensus. Single measure intraclass correlations were used to evaluate inter-rater reliability. In contrast to Pearson correlation, this measure also takes into account absolute rating score differences. Inter-rater correlations for the seven criteria ranged from 0.52 to 0.80 (single-measure intraclass correlation; Table 2). The criterion uni-vs multidimensional illness description, which showed an inter-rater correlation of $<0.60$, was omitted from further analyses. The mean score was calculated from the remaining six criteria. Inter-rater correlation for this summary scale was 0.79 (single-measure intraclass correlation).

Correlations between the six criteria were examined. While correlations with story coherence lay between 0.53 and 0.70 (Pearson $r$ ), the five other criteria showed greater correlations (0.81-0.92; Pearson $r$ ). The summary integration scale is used here. Where calculations using story coherence alone gave results that differed from calculations using the summary scale, the differences are indicated.

Illness factors (diagnosis, age at diagnosis, type and duration of therapy, incidence of complications, late effects) were assessed by reviewing hospital case notes. Sociodemographic factors (family status at age of illness, present social, educational and professional status) were assessed by a structured interview.
Two questionnaires were administered to the participants to evaluate illness-related distress:

- The Revised Impact of Event Scale (R-IES) [30] is a 15-item questionnaire that employs a four-point scale ("not at all" to "often") to assess symptoms of intrusion and avoidance evoked by a distressing experience. Examples of items assessing intrusion are: "I thought about it, when I didn't mean to" or "I had trouble falling asleep or staying asleep, because pictures or thoughts about it came into my mind". Examples of questions assessing avoidance are: "I tried to remove it from memory" or "I felt as if it hadn't happened or it wasn't real". Horowitz et al. [30] gave an internal consistency of 0.82 (Cronbach's a) for avoidance and 0.78 for intrusion. The retest reliability after an interval of 1 week was 0.79 (Pearson $r$ ) for avoidance and 0.89 for intrusion.

- A purpose-designed questionnaire, specifically developed for this study, included seven items (e.g. "Therapy was for me", "Thoughts of a relapse are for me") and used a four-point scale ("not at all distressing" to "very distressing") to measure distress retrospectively attributed to illness and therapy, and distress evoked by current memories of illness and therapy and by thoughts of a relapse.

Psychopathological symptoms were investigated with the Symptom Checklist 90-Revised (SCL-90-R) [31]. This 90-item questionnaire evaluates psychological distress on nine symptom dimensions (somatization, obsessive-compulsive, interpersonal sensitivity, depression, anxiety, hostility, phobic anxiety, paranoid ideation, psychoticism) and on a global severity index, using a fivepoint scale ("not at all" to "extremely"). Cronbach's a for the nine areas was between 0.79 and 0.89 in the German version used. A good retest reliability for subjects after an interval of 1 week is reported.

The Frankfurt Body Concept Scales (FBCS) [32] were used to measure different aspects of body image. This 64-item questionnaire comprises nine subscales: state of physical health, care of the body and of bodily functions, physical efficiency, body contact, sexuality, body self-acceptance, acceptance of the body by others, physical appearance, perception of body odour. Answers are given on a six-point scale, ranging from "not at all true" to "very true". Cronbach's a for the individual dimensions was between 0.46 and 0.93 . The retest reliability for the entire test was $0.62-0.93$ (intervals from 4 days to 3 months were tested).

Health locus of control represents the appraisal of experiences related to illness and health. It was examined with a standardized questionnaire (Fragebogen zur Erhebung von Kontrollüberzeugungen zu Krankheit und Gesundheit, KKG) [33] which contains 21 questions, using a six-point scale ("not at all true" to "very 
true"). Like the Multidimensional Health Locus of Control Scale devised by Wallston et al. [34], this German questionnaire consists of three scales: internality (illness and health are subject to personal acting and control), social externality (illness and health are subject to the influence of other people, e.g. doctors), and fatalistic externality (illness and health are subject to chance). Cronbach's a for the three scales were $0.64-0.77$, while the retest reliability was $0.74-0.78$

Procedures

The study protocol was approved by the Ethics Committee of Basel University Children's Hospital and written consent was obtained from each participant. Subjects who met the inclusion criteria received information about the study by mail and were contacted by a follow-up phone call to ask for participation. Interviews were conducted at the child psychiatry outpatient clinic, near the children's hospital. Assessment started with the illness narrative and was followed by the structured interview to gather socioeconomic data and the completion of three questionnaires. Two questionnaires (FBCS, KKG) were completed by the participants at home and sent back by mail. Nine patients (seven male, two female) did not return them.

Statistical analyses

One-sample $t$-tests were used to compare mean scores of the sample with corresponding SCL-90-R, FBCS, and KKG normative sample mean scores. Scores of means of distress attributed to illness and therapy-related experiences were compared using paired sample $t$-tests. The effects of potential explanatory factors on integration scores and the association between integration and present psychosocial condition were investigated with two-sample $t$-tests for binary factors and by Pearson bivariate correlations for normally distributed continuous factors, or Spearman bivariate correlations for continuous factors without normal distribution.

\section{Results}

\section{Descriptive findings}

Analysis of the narrative interviews showed great differences in the psychological integration of the experience of cancer. The distribution tended to be normal, covering a wide range from poor to very good integration. The degree of integration was not correlated with sex, illness factors (diagnosis, age at diagnosis, therapy duration or intensity), age at interview, or educational or professional status. However, there was a strong positive correlation between the criterion story coherence of the integration coding system and age at diagnosis (0.51, Pearson $r ; P<0.001)$ and a negative correlation between story coherence and time since end of therapy $(-0.30$, Pearson $r ; P<0.05)$. Thus, the older the subjects at the time of diagnosis and the shorter the period since the end of therapy, the more coherent were the narratives.

Table 3 Distress related to illness and therapy $(n=60)$

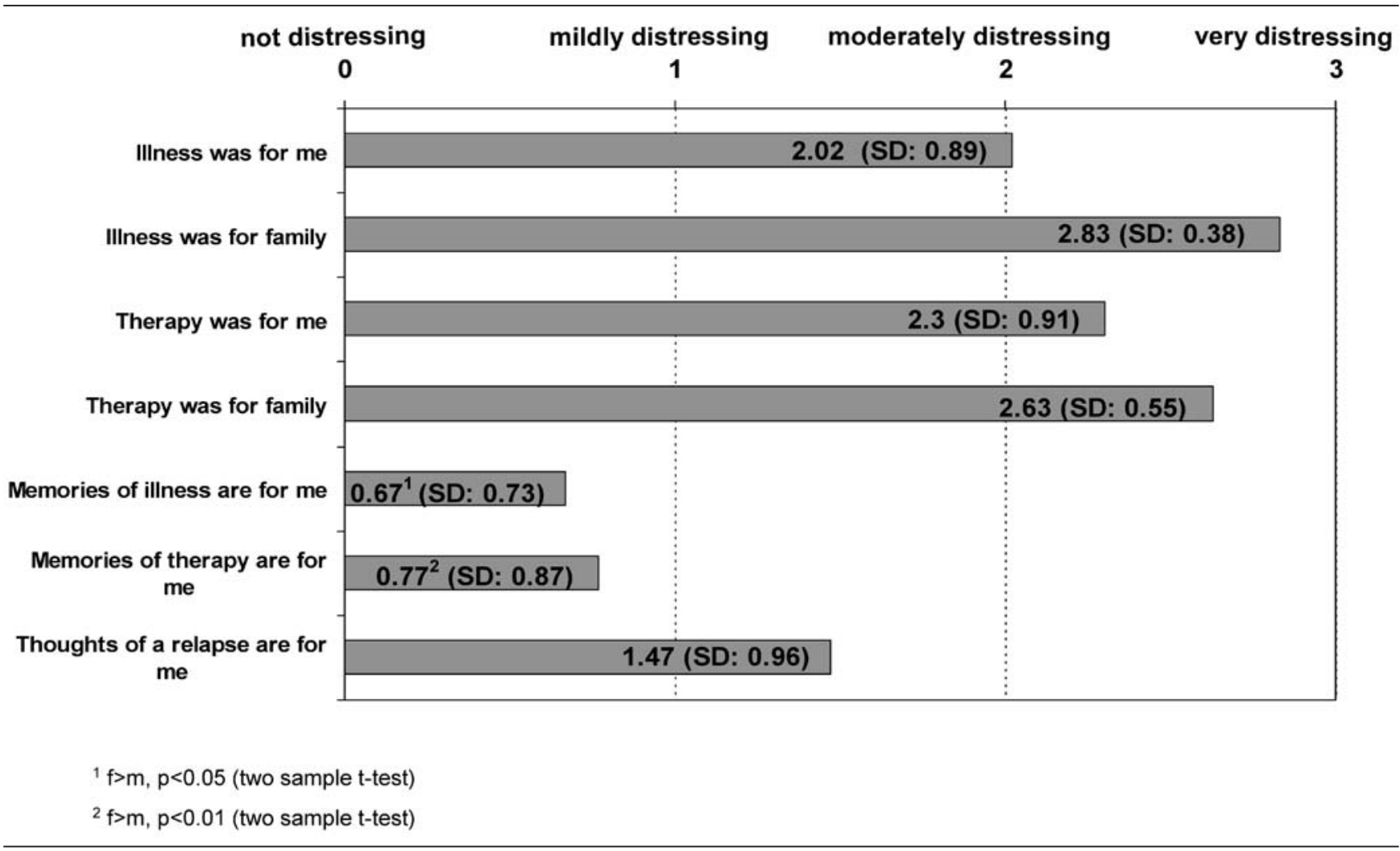


Table 4 Sample mean scores differing from SCL-90-R, FBCS and KKG norm mean scores (one-sample $t$-test)

\begin{tabular}{llcc}
\hline & Mean $(t$ value $)$ & SD & $P$ value \\
\hline SCL-90-R $(n=60)$ & 53.0 & 9.9 & 0.022 \\
Obsessive-compulsive & & & \\
FBCS $(n=51)$ & 70.7 & 27.5 & 0.000 \\
Care of the body and of bodily functions & & & \\
KKG $(n=51)$ & 53.6 & 12.7 & 0.047 \\
Internality & 55.2 & 10.6 & 0.001 \\
Social externality & & & \\
\hline
\end{tabular}

In the purpose-designed questionnaire, most subjects retrospectively attributed high levels of distress to their experience of illness and therapy (Table 3). Distress related to illness and therapy was judged as having been even greater for the family than for the patients themselves $(P<0.001$ for illness, $P<0.01$ for therapy; paired sample $t$-test). While subjects perceived the therapy as more distressing than the illness $(P<0.01$; paired sample t-test), they felt that for their family, the illness had been more distressing than the therapy $(P<0.01$; paired sample $t$-test). Current memories of illness and therapy were associated with little distress, and thoughts of a relapse with moderate distress. These findings were confirmed by the results of the R-IES that showed low mean scores for intrusion (3.92, SD 4.87) and avoidance (5.03, SD 7.55). Current memories of illness and therapy evoked significantly more distress in women than in men. There were no other differences between the sexes.

Mean scores of the standardized measures (SCL-90$\mathrm{R}$, FBCS, KKG) were compared with test norm scores for the general population. Few differences were identified. The childhood cancer survivors of this study group attributed a high value to care of the body and of bodily functions (Table 4).

Factors correlating with integration

While no association was found between integration and SCL-90-R or FBCS scores, there was an association between integration and health locus of control. Subjects with high integration scores, indicating good psychological integration, showed lower scores for fatalistic externality in the KKG $(-0.35$, Pearson $r ; P<0.01)$. In addition, there was a tendency to more internality and less social externality in participants with good integration, but these findings were not statistically significant.

Survivors with good integration perceived their illness retrospectively as more distressing than survivors with poor integration. Integration correlated positively with distress attributed to illness $(0.35$, Spearman rho; $P<0.01)$, distress attributed to therapy $(0.41$, Spearman rho; $P<0.001)$, and distress for the family attributed to therapy $(0.46$, Spearman rho; $P<0.001)$. In contrast, integration did not correlate significantly with distress evoked by current memories of illness and therapy or by thoughts of a relapse, nor with R-IES scores for intrusion and avoidance.

Of the 60 subjects, 38 (63.3\%) were married or living in a stable relationship. Good integration was associated with having a partner $(P<0.01$; two-sample $t$-test $)$.

\section{Discussion}

\section{Subjects and method}

This study was designed as an exploratory investigation. It was aimed at evaluating a concept of "integration" and the association between the psychological integration of illness experiences and psychological condition within an unselected sample of former childhood cancer patients. More than $80 \%$ of the subjects contacted agreed to take part in this retrospective study several years after ending therapy. This is a surprisingly high rate. Most subjects gave their desire to help children who would have cancer in the future as the reason for their participation. However, the high number of participants also indicates a great openness on the part of many survivors in dealing with their experiences. Narrative was selected as a research instrument because of its outstanding significance for the construction of identity and for the working through of psychological trauma. The high inter-rater correlations proved that narrative analysis is a reliable method to test integration. It is harder to evaluate the validity, the degree of accuracy, with which the analysis of narrative actually measures the psychological integration of the experience of illness and the significance that integration plays in the working-through process. This limitation is, however, a part of every exploratory investigation. It was the aim of this study to test hypotheses and questions which could be of importance for clinical psychooncological work. Narratives were only assessed at one time. Therefore, no statements can be made about the stability of this method. Longitudinal investigations 
with repeated assessments would be necessary to judge whether narrative quality is a stable measure for the psychological integration of stressful experiences.

In contrast to previous investigations [5, 9], the successful psychological integration of the experience of cancer was not defined as good coping; instead, the correlation between integration and psychological outcome was tested. Assessment of integration is one of many ways of analysing narratives. Like every method of interpretation, it is based on a theoretical model, in this case a psychodynamic definition of integrating psychological trauma, and is therefore limited in providing only a selective way of understanding narratives. The ability to express oneself verbally and to develop a narrative varies widely between individuals. That this factor influenced the evaluation of integration cannot be excluded. However, the comparison of integration with educational status and, possibly connected, the greater ability of participants with more education to express themselves verbally, did not confirm this possibility. Neither a positive nor a negative correlation was apparent.

\section{Descriptive findings}

The narrative interviews displayed a wide range of ways in which patients have psychologically integrated the experience of their illness. Some participants related highly coherent stories, rich in differentiated emotions and personal meaning that allowed the illness's influence on biography and relationships to be accepted. Other participants could only remember fragments, and struggled to give the story a coherent flow or to give coherence to their memories with emotions. Sometimes the interviewer had actively to keep the narrative going.

Illness and therapy were experienced as very distressing by the majority of patients. Many reported in the narratives that they had felt more affected by the painful and visible side effects of treatment than by the more abstract threat of illness. Conversely, patients stated that their families had suffered more from the illness and its potentially fatal outcome than from the therapy. In the narratives, a very high level of distress was particularly attributed to parents, who were also regarded by almost all patients as their greatest source of support during the illness. By the standardized measures used for assessment, participants showed hardly any differences from normative scores in psychopathology, health locus of control or body concept, and intrusive and avoidant symptoms were low. In considering these findings, it must be borne in mind that the study did not aim to evaluate the general psychological adjustment of childhood cancer survivors. This has been done in other studies with appropriate design, using larger and less-heterogeneous samples and including control groups.

\section{Factors correlating with integration}

Integration was not associated with any demographic or illness factors. This is in line with the findings of Boman and Bodegard [10], who studied coping, defined similarly to our term integration, and its relationship to factors associated with illness, treatment and demographic background in 30 survivors of childhood cancer. In their study, there was a tendency for coping to correlate with illness/treatment impairments and intellectual ability, but statistically significant correlations were only observed for diagnosis (patients with leukaemia and lymphoma showed better coping than did patients with solid tumours) and continuous complete remission.

Boman and Bodegard found no correlation between coping and age at illness onset. In the present study, the integration criteria that represented different aspects of emotional working through and expression did not correlate with age at diagnosis, while story coherence increased in patients who had been older at the time of illness onset. It may be concluded that conscious (explicit) memories play a leading role in the ability to create a formally coherent narrative, while emotional integration seems to be determined by multiple intrapsychic and interpersonal factors.

Integration did not correlate with measures of SCL90, R-IES and FBCS. There could be a number of reasons for this. First, it can be argued that the sample mean scores for the three questionnaires lay within the normal range and therefore differences would not be expected. Second, psychopathology and body concept are influenced by so many other factors not considered in this investigation, that the effect of integration of the illness experiences alone may not be significant enough to be distinguished. Third, there may be no association between integration and illness-related psychological outcome.

However, there were factors that correlated with integration. Individuals with good integration scored lower for fatalistic externality; that is, they saw chance as having less to do with illness and health. Individuals with good integration also experienced illness and therapy retrospectively as more distressing than did those with poor integration, although the review of their clinical records did not reveal any significant differences in diagnosis, duration or intensity of therapy, or severity of complications. On the other hand, there was no correlation between good integration and a greater level of distress evoked by current memories of illness and therapy or thoughts of a relapse. These results could indicate that those people who succeeded in integrating their illness experiences have a greater ability to accept painful feelings and to allow them to emerge, as well as a greater readiness to accept responsibility for health and medical care, and probably also a greater level of autonomy in relation to the family. The positive correlation between in- 
tegration and having a partner could support the last suggestion, with the limitation that this finding emerged from the exploratory analysis rather than from hypothesis testing.

It is not clear whether successful integration is responsible for the development of autonomy and for the acceptance of stressful experiences as part of one's personal biography, or whether these parameters precede integration. However, many survivors found integration helpful in working through their illness experiences, as shown by the high percentage of survivors who agreed to take part in the study and talk about their experiences, and by the answers to a questionnaire sent by mail to participants $12-18$ months after the interviews. In this follow-up assessment, they were asked how the interview had affected their ability to cope with illness and therapy. Of the 42 subjects who replied, two-thirds stated that the interview had had a positive influence and onethird stated that it had had no influence on subsequent coping. None of the subjects attributed a negative effect to the interview.

The findings of this exploratory study suggest that it may be an important clinical task, helping young cancer patients and their families to create and maintain their personal narratives of illness and treatment. The provision of this support is not limited to psychosocial workers, though. Through continuous information and care, listening to anxieties and being witnesses to their suffering, all professionals can help patients to bring their experiences into a form that can be comprehended by the patient, communicated and shared [21, 35]. However, despite the importance for psychological adjustment of integrating stressful experiences, it should be remembered that the process of coping is always individual. For some people and in certain situations other strategies, such as denial, may prove to be more effective. The natural course of integration and the effects on adjustment of psychological interventions that support integration can only be evaluated by prospective trials.

Acknowledgements We are grateful to all participants for talking openly about their experiences. We thank Rosemarie MeierHunzinger, whose help was indispensable in the realization of this study, and Kai von Klitzing and Agnes von Wyl for critical revision of the manuscript. This research was funded by the De Bernardi-Stiftung Basel and the Stiftung der Elternvereinigung krebskranker Kinder Regio Basiliensis.

\section{References}

1. Mulhern RK, Wasserman AL, Friedman AG, Fairclough D (1989) Social competence and behavioral adjustment of children who are long-term survivors of cancer. Pediatrics 83:18-25

2. Gray RE, Doan BD, Shermer P, FitzGerald AV, Berry MP, Jenkin D, et al (1992) Psychologic adaptation of survivors of childhood cancer. Cancer 70:2713-2721

3. Varni JW, Katz ER, Colegrove R, Dolgin M (1994) Perceived stress and adjustment of long-term survivors of childhood cancer. J Psychosoc Oncol 12:1-16

4. Elkin TD, Phipps S, Mulhern RK, Fairclough D (1997) Psychological functioning of adolescent and young adult survivors of pediatric malignancy. Med Pediatr Oncol 29:582-588

5. Fritz GK, Williams JR, Amylon M (1988) After treatment ends: psychosocial sequelae in pediatric cancer survivors. Am J Orthopsychiatry 58:552-561

6. Madan-Swain A, Brown RT, Sexon SB, Baldwin K, Pais R, Ragab A (1994) Adolescent cancer survivors: psychosocial and familial adaptation. Psychosomatics 35:453-459
7. Shroff Pendley J, Dahlquist L, Dreyer Z (1997) Body image and psychosocial adjustment in adolescent cancer survivors. J Pediatr Psychol 22:29-43

8. Mäkipernaa A (1989) Long-term quality of life and psychosocial coping after treatment of solid tumors in childhood. Acta Paediatr Scand 78:728-735

9. Boman K, Bodegard G (1995) Psychological long-term coping with experience of disease and treatment in childhood cancer survivors. Acta Paediatr 84:1395-1402

10. Boman K, Bodegard G (2000) Longterm coping in childhood cancer survivors: influence of illness, treatment and demographic background factors. Acta Paediatr 89:105-111

11. Bauld C, Anderson V, Arnold J (1998) Psychosocial aspects of adolescent cancer survival. J Paediatr Child Health 34:120-126

12. Kazak AE (1994) Implications of survival: pediatric oncology patients and their families. Pediatric psychooncology. Oxford University Press, New York

13. Eiser C (1998) Practitioner review: long-term consequences of childhood cancer. J Child Psychol Psychiatry 39:621-633

14. Conrad P (1990) Qualitative research on chronic illness: a commentary on method and conceptual development. Soc Sci Med 30:1257-1263
15. Puukko L-RM, Hirvonen E, Aalberg V, Hovi L, Rautonen J, Siimes MA (1997) Sexuality of young women surviving leukaemia. Arch Dis Child 76:197-202

16. Janet P (1973) L'automatisme psychologique: essai de psychologie expérimentale sur les formes inférieures de l'activité humaine. Société Pierre Janet/Payot, Paris

17. Van der Kolk BA, van der Hart O (1989) Pierre Janet and the breakdown of adaptation in psychological trauma. Am J Psychiatry 146:1530-1540

18. Spiegel D (1999) Healing words: emotional expression and disease outcome. JAMA 281:1328-1329

19. Antonovsky A (1981) Unraveling the mystery of health. How people manage stress and stay well. Jossey-Bass, San Francisco

20. Janet P (1983) L'état mental des hystériques. Lafitte Reprints, Marseilles

21. Polkinghorne DE (1991) Narrative and self-concept. J Narrative Life History $1: 135-153$

22. Kohler Riessman C (1990) Strategic uses of narrative in the presentation of self and illness: a research note. Soc Sci Med 30:1195-2000 
23. McCabe A, Peterson C (1991) Structure as a way of understanding (preface). Developing narrative structure. Lawrence Erlbaum, Hillsdale

24. Elwyn G, Gwyn R (1999) Stories we hear and stories we tell: analysing talk in clinical practice. BMJ 318:186-188

25. Kohler Riessman C (1989) Life events, meaning and narrative: the case of infidelity and divorce. Soc Sci Med 29:743-751

26. Viney LL, Bousfield L (1991) Narrative analysis: a method of psychosocial research for AIDS-affected people. Soc Sci Med 32:757-765
27. Cohler BJ (1991) The life story and the study of resilience and response to adversity. J Narrative Life History $1: 169-200$

28. Greenhalgh T, Hurwitz B (1999) Why study narrative? BMJ 318:48-50

29. Oppenheim D, Wamboldt FS, Gavin LA, Renouf AG, Emde RN (1996) Couples' co-construction of the story of their child's birth: associations with marital adaptation. J Narrative Life History 6:1-21

30. Horowitz M, Wilner N, Alvarez W (1979) Impact of Event Scale: a measure of subjective stress. Psychosom Med 41:209-218

31. Derogatis LR (1983) Symptom Checklist-90-R manual. Clinical Psychometric Research, Towson, MD

32. Deusinger IM (1998) Die Frankfurter Körperkonzeptskalen (Frankfurt Body Concept Scales). Hogrefe, Goettingen
33. Lohaus A, Schmitt GM (1989) KKG, Fragebogen zur Erhebung von Kontrollüberzeugungen zu Krankheit und Gesundheit (Multidimensional Health Locus of Control Scale-German modified version). Hogrefe, Goettingen

34. Wallston KA, Wallston BS, De Vellis R (1978) Development of the Multidimensional Health Locus of Control (MHCL) Scales. Health Educ Monographs 6:160-170

35. Jones AH (1999) Narrative in medical ethics. BMJ 318:253-256 